Open Access to Pharmaceutical and Medical Research

(C) 2011-18, publisher and licensee JDDT, This is an Open Access article which permits unrestricted non-commercial use, provided the original work is properly cited

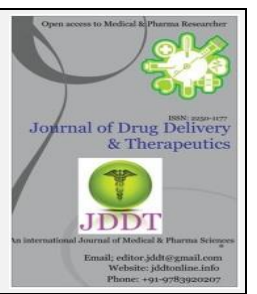

Open $\odot$ Access

Review Article

\title{
Dendrimers as a Novel Carrier in Anti-HIV Therapy
}

\author{
P. Arshada, P. Dineshkumara*, K. Naga Jyothia, M. Karthika, Govindaraj Saravanan ${ }^{b}$ \\ A-Research Center in Pharmaceutics, Hindu College of Pharmacy, Acharya Nagarjuna University, Guntur, Andhra Pradesh, India. \\ B- Department of Pharmaceutical Chemistry, MNR College of Pharmacy, Fasalwadi, Sangareddy-502294, Telangana, India
}

\begin{abstract}
The present treatments for HIV transfection include chemical agents and gene therapies. Although many chemical drugs, peptide s and genes have been developed for HIV inhibition, a variety of non-ignorable drawbacks limited the efficiency of these materials. Dendrimers has ability to carrier of antiviral drugs due to some properties such as mono-dispersity, defined structure, amenability for functionalization using diverse ligands and its low-nanometer size. In this review, we discuss the application of dendrimers as both therapeutic agents and non-viral vectors of chemical agents and genes for HIV treatment. In one way, dendrimers with functional end groups combine with the gp120 of HIV and CD4 molecule of host cell to suppress the attachment of HIV to the host cell. In another way, dendrimers are also able to transfer chemical drugs and genes into the host cells, which increase the anti-HIV activity of these materials. Dendrimers as therapeutic tools provide a potential treatment for HIV infection.
\end{abstract}

Keywords: Dendrimers, Drug release, Drug targeting, gp120, CD4, Antiviral drug

Article Info: Received 10 July 2019; Review Completed 23 Aug 2019; Accepted 28 Aug 2019; Available online 22 Oct 2019

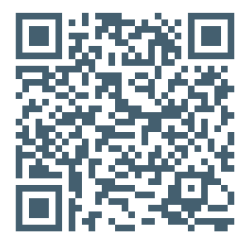

\section{Cite this article as:}

Arshad P, Dineshkumar P, Naga Jyothi K, Karthik M, Saravanan G, Dendrimers as a Novel Carrier in Anti-HIV Therapy, Journal of Drug Delivery and Therapeutics. 2019; 9(5-s):195-200 http://dx.doi.org/10.22270/jddt.v9i5-s.3650

*Address for Correspondence:

Andhra Pradesh

Dr. P. DINESH KUMAR M.PHARM, PhD, Research Center of Pharmaceutics, Hindu College of Pharmacy, Amaravathi road, Guntur,

\section{INTRODUCTION}

Dendrimers are macromolecules with a defined globular shape that were synthesized for the first time at the end of the 1970s. They are sophisticated and curious molecules and have attracted the interest of chemists owing to their potential applications in different areas, such as catalysis, materials science and biomedicine[1].One of the most attractive applications in the biomedical field is the use of functionalized dendrimers as antiviral agents. These dendrimers are able to form stable complexes with viral structures or receptors at the cell surface, resulting in disruption of the virus-cell interaction during the infection process. Dendrimers have emerged as important tools for drug discovery because of their ease of surface modification as well as their ability to interact with charged functional groups. This review suggest that dendrimers may have applications in prion infections. They are also very efficient anti-virals, acting by both inhibiting cell entry and preventing replication of pathogens. They have shown immense potential to act against HIV. The life cycle of HIV comprises a number of steps: first, HIV attach to the host cell and fuses with the membrane; then, the viral RNA reverse transcripts to form
DNA and integrates into the host cell's DNA; next, mRNA is formed via transcription and protein expresses; finally, the new virus is assembled automatically. Each of the steps could be considered as the target for intervention [2]. A lot of polymers, chemical agents and gene therapies are widely used to block these two steps for HIV replication, yet most of the treatments are lacking in effectiveness [3]. The dendrimers have been designed with specific functional end groups to preferentially interact with the envelope protein of HIV and receptors on the host cells in order to suppress the combination between HIV and host cells and later stages of HIV replication [4]. The dendrimers with large amounts of peripheral groups and interior cavities are potential vectors for chemical drugs, peptides and genes for HIV inhibition. These materials are capable to either interacting with the peripheral groups or be encapsulated into the cavities of dendrimers with hydrogen bonds, electrostatic and hydrophobic interactions $[5,6]$. Other anionic polymers acting on target HIV, and to the nucleotide or non-nucleoside reverse transcriptase inhibitors or the integrate inhibitors acting on intracellular targets $[7,8,9,10]$. 


\section{Antiviral activity of dendrimers}

Dendrimers used as antiviral agents has been one of the most successful avenues in dendrimer-based drug discovery. Dendrimers either prevent binding of viruses to the target cell surface or prevent replication of the viral genome. The ability to prepare a diverse array of dendrimers, patterns of biological activity can be investigated where subtle changes in the dendrimer design parameters type of initiator, branching unit type, dendrimer generation, linker, and surfaces may have a bearing on the biological properties of the individual dendrimers.

\section{Prevention of virus binding to the target cell surface}

Multiple carbohydrate binding proteins present on viruses play a critical role in invasion of the host cell. Carbohydrates present on the target cell surface can be linked to various dendrimers, which can act as receptors for viruses. Thus, glycodendrimers with carbohydrates at the periphery were tested for their ability to prevent binding of the influenza virus to host cell surface carbohydrates.[11,12] Carbosilane dendrimers functionalized with sialyllactose (a trisaccharide) were also found to be effective inhibitors of influenza hem agglutinin induced agglutination of erythrocytes.[13] The non-cytotoxic polysulfated galactose derivatised poly(propyleneimine) dendrimers inhibit the infection of laboratory-isolated HIV-1 as efficiently as dextran sulfate.[14] Sulfonated polylysine dendrimer with a benhydrylamine core has been shown to inhibit viral absorption[15]. The anti-HIV activity of dendrimers and glycodendrimers is being extensively studied. A recent study indicated that PPI and manosylated PPI dendrimer alone possess some anti-HIV activity. These nanocarrier systems are being explored further for controlled and targeted delivery of anti-HIV drugs[16].

\section{Dendrimer based drug targeted approach}

Dendrimers highly branched nanocarriers. The surfaces of dendrimers can be easily functionalized due to the availability of multiple functional groups. Dendrimers have been investigated for the delivery of drugs across the BBB. Polyether-co-polyester dendrimers containing the cytotoxic drug methotrexate were conjugated to Dglucosamine to enhance the BBB permeability in order to deliver high amounts of drug to the CNS[17]. In another study, lamivudine-loaded mannosylated PAMAM dendrimers were evaluated for their in vitro antiretroviral activity in HIV-infected MT2 cells. It was observed that following encapsulation within dendrimers, lamivudine exhibited greater antiretroviral activity as a result of 21fold higher drug uptake when compared to drug in solution [17].In this study designed PAMAM having PEG grafts as a novel drug carrier. PEG were combined to essentially every chain of dendrimers with the generation G4 and G5. Moreover we prepared the PEGylated PAMAM dendrimers encapsulating the anti-HIV drug lamivudine. While encapsulating ability of PEGylated PAMAM dendrimers are increased when compared with nonPEGylated PAMAM dendrimers. Performed drug release studies of PEGylated PAMAM dendrimers showed prolong drug release for longer time when compared with nonPEGylated PAMAM dendrimers[18]. In this study successfully synthesized and conjugated PEGylated PAMAM dendrimers with anti HIV drug stavudine. Hemolytic toxicity studies were carried out for PEGylated PAMAM dendrimers which showed that haemolysis of cell was very negligible in PEGylated PAMAM dendrimers[19]. 5.0 G EDA PAMAM dendrimers were synthesized and
PEGylated using PEG 600 using epichlorhydrin as crosslinking agent. PEGylated 5.0 G PAMAM dendrimers loaded with Efavirenz (EFV) the results it is proved that PEGylated 5.0 G PAMAM-EFV dendrimers have shown prolonged drugrelease property [20].

\section{Glycodendrimers}

These are dendrimers presenting at their surface multiple copies of carbohydrates, have been recently developed and are excellent tools with which to address carbohydrateprotein interactions in biological processes at the molecular level and also to study the multivalent effect [21]. Carbohydrates confer to these systems high selectivity to interact with specific lectin receptors; however, until recently, few glycodendrimers have found biomedical applications. For instance, one of these applications enables these molecules to interact with Fimbrae protein to avoid infection by Escherichia coli[22]. Another interesting example is a fourth-generation PAMAM dendrimer conjugated with sialic acid.[23,24] These sialic acids present at the dendrimer surface are able to interact with haemagglutinin, the major surface glycoprotein of influenza A virus, and prevent viral adhesion to target cells exhibiting sialic acid at their surfaces. This activity has been tested in vitro and in vivo using a murine influenza pneumonitis model [25].To the best of our knowledge, this is the only example described of glycodendrimers as antiviral drugs. In this one, the main antiviral strategy so far has been based on the binding of the dendrimer to the viral surface. However, many viruses, such as influenza A virus or HIV, present a high degree of mutation leading to major changes in the envelope protein glycosylation patterns when comparing the different strains. This is one of the reasons why the development of a viral vaccine is so elusive in these particular cases. The glycopeptide dendrimers have been used in several ways such as antitumor and antiviral prophylactic or therapeutic vaccines[21].

\section{Prevention of HIV Attachment to the Surface of Host Cells}

\section{CD4 Receptors}

HIV infection starts with attachment of envelope protein gp120 to CD4 molecules on the host cell surface, followed by engagement of chemokine receptors (CXCR4 and CCR5) to form a CD4-gp120-chemokine receptor complex. Subsequently, envelope protein gp41 of the virus mediates the membrane fusion [26]. The dendrimers with functionalized end groups were present that were developed to combine with gp120 or CD4 molecules to impede the subsequent steps of HIV infection. The discovery of the mechanism of HIV inhibition, two polyanionic dendrimers BRI2932 (SPL2923) and BRI6195 (SPL6195) was found to inhibit the replication of HIV (strain IIIB) at an EC50 of 0.1 and $0.3 \mu \mathrm{g} / \mathrm{mL}$ respectively, with extremely low cytotoxicity to the host cells. A gp120 binding assay and virus adsorption assay indicated that both compounds have an effect on the docking of HIV to the host cells. The SPL2923 at higher concentration could also block the later stages of HIV infection. Correspondingly, results from cellular uptake experiments testified that SPL2923 was capable of invading the host cell, whereas SPL6195 was not [27].

\section{DC-SIGN Receptors}

DC-SIGN receptor is a tetrameric C-type lectin mainly presented on the surface of immature dendritic cells (DCs) for modulating the immune response against pathogen 
$[28,29]$. In previous studies, DC-SIGN was testified as the receptors of a number of pathogens, including Ebola, simian immuno-deficiency virus (SIV), HIV and cytomegalovirus. The mechanism of DC-SIGN to act as a receptor had been exploited with high affinity for mannose glycans moieties on the pathogens $[30,31,32]$. For instance, HIV possesses a high density of the Man- $\alpha 1,2-$ Man motif on the gp120 [33]. Thus, a nucleic acid-encoded carbohydrate library that focused on mannose and its derivatives suggested that an assembly of mannose with an aryl group at the 6-position of the terminal unit is beneficial for DCSIGN binding. The binding affinity of the assemblies to DCSIGN was measured and the assembly with highest affinity was covalently bound to PAMAM. The studies on the assembly modified PAMAM showed intensive inhibition on the interaction between gp120 and DCs at $10 \mu \mathrm{M}(60 \mu \mathrm{M}$ in mannose), whereas a natural polymer of mannosemannan only partially inhibited the interaction at $500 \mu \mathrm{M}$ in mannose [34].

\section{Glycosphingolipids (GSLs)}

In non-CD4-expressing cells the potential binding receptors of HIV have been supposed to be GSLs [35,36]. It has been verified by several studies that the gp 120 of HIV virus would bind with the GSLs for cell entrance [37]. the dendrimer-based antagonists for HIV could be changed with carbohydrates moieties to mimic the local spatial structure of GSLs. Galactose [38], cellobitose [39,40], globotriose and 3'-sialyllactose [41] have been employed as functional dendrimer end groups to suppress attachment of HIV to host cells. In conformity with the previous compounds, both of the galactose and cellobitose were sulfated to achieve effective inhibition activity.

The interactions between particular sulfate and V3 loop of gp120 was confirm by Richard and colleagues [42], implementation of the inhibition of gp120 adherence to immobilized sulfatide by galactosyl ceramide (GalCer), sulfatide galactosyl ceramide (SGalCer), ceramide, dextran sulfate (DxS), and chondroitin sulfate (ChS). The previous studies showed that GalCer and its 3-sulfated derivativeSGalCer had high affinity to gp120 which mediated the HIV-1 infection, and a series of analogs of GalCer-modified dendrimers showed impressive anti-HIV activity [43, 44]. The galactose moieties were modified on a polypropylenimine tetrahexacontaamine dendrimer (PS Gal 64mer). The dendrimer with specific position C-3 sulfate modified galactose, akin to SGalCer, was certified by the HIV inhibition [45]. The positions were compared with the position-specifically sulfated galactose modified dendrimer, randomly sulfated cellobitose was connected to a generation 3 polylysine dendrimer (PLDG3). The antiHIV activity assay demonstrated that PLDG3 had less antiviral activity than dextran sulfate, curdlan sulfate and azidothymidine (AZT), but similar activity to dideoxycytidine (ddC). The low molecular weight of oligosaccharides (such as cellobitose) have little or no antiHIV activity [46], the high biological activity of PLDG3 was ascribed to the cluster effects of sulfated cellobitose on PLDG3 [47]. In addition, the sulfated cellobitose functional group was also attached to an amphiphilic lysine dendrimer (SCSLD3), which was covalently bound to a stearylamide. The long stearyl chains were interact with the HIV lipid bilayer and destroy the HIV lipid bilayer, similarly to the function of surface active agents with both hydrophilic (sulfated cellobitose) and hydrophobic ends (stearyl chain) [48]. The preliminary anti-HIV activity of SCSLD3 was determined to have as high activity as PLDG3 [49].

\section{Prevention of Replication of HIV in Host Cells}

\section{Polycationic Dendrimers}

Unmodified polycationic dendrimers-PAMAM have been developed to inhibit binding of Tat protein to trans-acting responsive element (TAR) RNA of HIV [50,51]. The cationic molecules were also including polyamine acridine based compounds [52] and polyallylamine hydrochloride (PAH) [53], which interacted with TAR RNA through electrostatic forces. A kind of polycationic based dendrimer with excellent activity against replication of HIV-1 was developed. The dendrimers were inhibited HIV-1 replication in MT-4 cells while they showed no inhibitory activity in human PBMC. The distinct anti-HIV activities were in line with the expression of heparan sulfate in the host cells. Thus, the inhibition of HIV replication was ascribed to the block of interactions between virus and heparan sulfate on the cell surface. This assumption was testified by the detection of charge distance of dendrimer moieties and heparan sulfate disaccharide unit. Besides, the dendrimers with spheroidal structure exhibited higher inhibition activity than those with comb-branched structures [54].

\section{Polyanionic Dendrimers}

Polyanionic dendrimers were also inhibits the HIV. The first developed compound SPL2923 has been found to have an effect on the reverse transcription process at a concentration of $100 \mu \mathrm{g} \mathrm{mL}^{-1}$ (5-fold of the concentration used for inhibiting the attachment to host cells). However, SPL 6195 could not penetrate into the host cells and interfere with the later stages of HIV infection, even if the concentration of the compound was increased 5-fold. It was reasonable to conclude that the differences on the core structure and peripheral groups of dendrimers definitely affected their capability to restrain the replication of HIV [55]. The SPL7013 and SPL7115 were inhibits the HIV-1 virus, and the results suggested that SPL7013 was capable of inhibiting the activity of reverse transcriptase (RT) of HIV-1.

\section{Dendrimers Acting as Potential Vectors for Drugs and Genes}

The anti-HIV chemical drugs have shown broad treatment failure and adverse effects which are related to their low cytotoxicity and high plasma concentration of drugs. Dendrimers as an ideal carrier could effectively bring the anti-HIV drugs into HIV-infected cells and take the place of virus to transfer genes into nucleus of HIV-infected cells in order to effectively interfere with the replication of HIV

\section{Dendrimers as Carriers for Chemical Drugs}

The chemical anti-HIV drugs widely used for inhibition of reverse transcription are divided into three categories: nucleoside analogue reverse transcriptase inhibitors (NRTI), non-nucleoside reverse transcriptase inhibitors (NNRTI) and protease inhibitors (PI). Efavirenz (EFV), lamivudine (3TC), zidovudine (AZT) and didanosine (ddI) are the most studied anti-HIV drugs due to their most favorable resistance profiles. Except for EFV, the others are all NRTIs. As monocytes/macrophages (Mo/Mac) were supposed to be the reservoir of HIV and played an important role on dissemination of virus throughout the body, antiretroviral drugs were expected to specifically intrude the Mo/Mac [56]. Lectin receptors were reported to be presented on the surface of the Mo/Mac $[57,58]$. Thus, connected with saccharides was supposed to be the most efficient way for drugs to get into Mo/Mac via combination of saccharides and lectin receptors $[59,60]$. 


\section{Dendrimers as Carriers for Genes}

Gene therapies for HIV inhibition can be divided into two categories: (1) oligodeoxynucleotides (ODNs) involving the HIV polypurine tract element mRNA (PPT), HIV antitransactivation responsive gene (TAR), HIV gene expression modulator 91 (GEM 91), HIV replication mRNA (REV) and a random mix of two of the ODNs to form a cocktail; (2) siRNA involving three sequence: siP24, siGAG1, siNEF and cocktails. The genes were transferred into cells via viral vectors. However, the use of viral vectors suffered from several defects. The viral vectors would induce severe immune reactions. The genes would bind non-specifically to serum proteins which decreased the bioactivity and induced toxic effect [61]. Thus, a new non-viral vector was required to play the role of gene carriers.

\section{Oligodeoxynucleotides (ODNs)}

The first to employ 2G-NN8 and 2G-NN16 as carriers for ODNs to form dendriplexes. The studies showed that the dendriplexes were able to protect ODNs from binding to both bovine serum albumin (BSA) and human serum albumin (HSA). The dendriplex formed by 2G-NN16 and TAR was shown by confocal microscopy to be internalized into the PMBC, and the ODNs alone showed non-sequence specific inhibition effects on PBMC and lymphocyte T-cells (MT-2), however, the dendriplexes exhibited sequence specificity in HIV inhibition to some extent [63].

\section{SiRNA}

As a carrier for siRNAs, 2G-NN16 provided a strong protection for siRNA challenged with heparin and ribonuclease (RNase). The dendriplexes was implemented on both Sup T1 cells and HIV-infected PBMC. Interestingly, naked siRNA showed successful uptake by Sup T1 cells, but was unable to enter the HIV-infected PMBC during the first 3 hours. The dendriplexes shows the highest transfection efficiency at a 2G-NN16/siRNA ratio of 1 or 2 . The higher + /- charge ratios would inhibit the viability of cells. The naked siRNA showed sequence-specific and concentrationdependent activity against the housekeeping gene (GAPDH) in uninfected SupT1 cells and HIV replication in infected PMBC via electroporation. The dendriplexes was tested directly on cells. The results indicated that the dendriplexes possessed higher inhibition activity on GADPH in SupT1 cells and HIV-infected PBMC than naked siRNAs, especially with the cocktails of siRNA [63]. Carbosilane dendrimer, polycationic dendrimer PAMAM and phosphorus-containing dendrimer- $\mathrm{G} 4\left(\mathrm{NH}+\mathrm{Et}_{2} \mathrm{Cl}^{-}\right)_{96}$ had been developed as carrier for gene therapies as well. The PAMAM and siRNAs, especially the cocktails, formed dendriplexes that not only exhibited conspicuous inhibition of HIV replication in vitro, but also effectively suppressed HIV infection in viremic RAG-hu mice[63].

\section{CONCLUSION}

The current status of dendrimer-based therapies for the treatment of HIV infection, and highlight the potential role of dendrimers as both therapeutic agents and non-viral vectors. As dendrimers have a lot of peripheric active groups, modified dendrimers have the ability to specifically combine with gp120 or CD4 molecule in order to block the attachment of HIV to the host cell. The application of dendrimers as carriers of chemical agents and genes is impressive. The dendrimers improve the efficiency of cellular uptake and anti-HIV activity on host cells, while the anti-HIV activity of the dendrimer-based formulations is not perfectly in accordance with the cellular uptake results. Hence, it's concluding that the release profile of drugs and the degradation ability of dendriplexes play an important role for anti-HIV activity. In conclusion, the explanation of dendrimer based drug delivery form will help in ART therapy by minimizing the drug resistance of anti-viral molecules. The dendrimers with specific functional groups offer significant benefits to prevent HIV infection.

\section{REFERENCES}

1. Matthews, O. A., Shipway, A. N. \& Stoddart, J. F. (1998). Dendrimers-branching out from curiosities into new technologies. Progress in Polymeric Science 23, 1-56.

2. De Clercq, E. Antiviral therapy for human immunodeficiency virus infections. Clin. Microbiol. Rev. 1995, 8, 200-239.

3. Fréchet, J.M.; Tomalia, D.A. Dendrimers and Other Dendritic Polymers; Wiley: New York, NY, USA, 2001.

4. Jiang, Y.-H.; Emau, P.; Cairns, J.S.; Flanary, L.; Morton, W.R.; McCarthy, T.D.; Tsai, C.-C. SPL7013 gel as a topical microbicide for prevention of vaginal transmission of SHIV89. 6P in macaques. AIDS Res. Hum. Retroviruses 2005, 21, 207-213.

5. Svenson, S.; Tomalia, D.A. Dendrimers in biomedical applications-Reflections on the field. Adv. Drug Deliv. Rev. 2005, 57, 2106-2129.

6. D'Emanuele, A.; Attwood, D. Dendrimer-Drug interactions. Adv. Drug. Deliv. Rev. 2005, 57, 2147-2162.

7. Grant RM, Hamer D, Hope T, et al. Whither or wither microbicides? Science. 2008;321(5888):532-534.

8. Vanpouille C, Arakelyan A, Margolis L. Microbicides: still a long road to success. Trends Microbiol. 2012;20(8):369-375.

9. Fichorova RN, Tucker LD, Anderson DJ. The molecular basis of nonoxynol9-induced vaginal inflammation and its possible relevance to human immunodeficiency virus type 1 transmission. J Infect Dis. 2001;184(4): 418-428.

10. McCormack S, Ramjee G, Kamali A, et al. PRO2000 vaginal gel for prevention of HIV-1 infection (Microbicides Development Programme 301): a phase 3, randomised, double-blind, parallel-group trial. Lancet. 2010;376(9749):1329-1337.

11. Tsvetkov DE et al. Neoglycoconjugates based on dendrimeric poly(aminoamides). Bioorg Khim 2002; 28: 518-534.

12. Roy R. Synthesis and some applications of chemically defined multivalent glycoconjugates. Curr Opin Struct Biol 1996; 6: 692-702.

13. Matsuoka $\mathrm{K}$ et al. Sugar chain-containing carbosilane dendrimer compounds, process for producing the same verotoxin neutralizers and antiviral agents. US Patent 2004/0040554, 2004.

14. Kensinger RD et al. Novel polysulfated galactose-derivatized dendrimers as binding antagonists of human immunodeficiency virus type 1 infection. Antimicrob Agents Chemother 2004; 48: 1614-1623.

15. Gong $Y$ et al. Evidence of dual sites of action of dendrimers: SPL-2999 inhibits both virus entry and late stages of herpes simplex virus replication. Antiviral Res 2002; 55: 319-329.

16. McCarthy TD et al. Dendrimer as drug: discovery and preclinical and clinical development of dendrimer-based microbicides for HIV and STI prevention. Mol Pharm 2005; 2: 313-318.

17. Torchilin VP. Recent advances with liposomes as pharmaceutical carriers. Nat Rev Drug Discov. 2005;4:14560. - A comprehensive review on liposomal brain drug delivery. [PubMed] [Google Scholar]

18. P. Dinesh Kumar, P.Vijayaraj Kumar, T. Panneer Selvam and K.R.S. Sambasiva Rao "Prolonged Drug Delivery System of PEGylated PAMAM Dendrimers with a Anti-HIV Drug" Research in Pharmacy 2013 ,3(2): 08-17. 
19. P. Dinesh Kumar, P.Vijayaraj Kumar, T. Panneer Selvam and K.R.S. Sambasiva Rao, "PEG Conjugated PAMAM Dendrimers with a AntiHIV Drug Stavudine for prolong release", Research in Biotechnology, 2013,4(2): 10-18.

20. Suneela Pyreddy, Pandurangan Dinesh Kumar, Palanirajan Vijayaraj Kumar, "Polyethylene glycolated PAMAM dendrimers-Efavirenz conjugates", International Journal of Pharmaceutical Investigation , 2014, 4 (1).

21. Bezous ka, K. Design, functional evaluation and biomedical applications of carbohydrate dendrimers (glycodendrimers). Reviews in Molecular Biotechnology ,(2002), 90, 269-90.

22. Nagahori, N., Lee, R. T., Nishimura, Inhibition of adhesion of type 1 fimbriated Escherichia coli to highly mannosylated ligands. ChemBioChem, (2002),3, 836-44.

23. Reuter, J. D., Myc, A., Hayes, Inhibition of viral adhesion and infection by sialic-acid-conjugated dendritic polymers. Bioconjugate Chemistry (1999) ,10, 271-8.

24. Landers, J. J., Cao, Z., Lee, Prevention of influenza pneumonitis by sialic acid-conjugated dendritic polymers. Journal of Infection Diseases, (2002), 186, 1222-30.

25. Wyatt, R.; Sodroski, J. The HIV-1 envelope glycoproteins: Fusogens, antigens, and immunogens. Science 1998, 280 1884-1888.

26. Witvrouw, M.; Fikkert, V.; Pluymers, W.; Matthews, B.; Mardel, K.; Schols, D.; Raff, J.; Debyser, Z.; De Clercq, E.; Holan, G. Polyanionic (i.e., polysulfonate) dendrimers can inhibit the replication of human immunodeficiency virus by interfering with both virus adsorption and later steps (reverse transcriptase/integrase) in the virus replicative cycle. Mol. Pharmacol. 2000, 58, 1100-1108.

27. Hug, P.; Lin, H.-M.J.; Korte, T.; Xiao, X.; Dimitrov, D.S.; Wang, J.M.; Puri, A.; Blumenthal, R. Glycosphingolipids promote entry of a broad range of human immunodeficiency virus type 1 isolates into cell lines expressing CD4, CXCR4, and/or CCR5. J. Virol. 2000, 74, 6377-6385.

28. Puri, A.; Hug, P.; Jernigan, K.; Barchi, J.; Kim, H.-Y.; Hamilton, J.; Wiels, J.; Murray, G.J.; Brady, R.O.; Blumenthal, R. The neutral glycosphingolipid globotriaosylceramide promotes fusion mediated by a CD4-dependent CXCR4-utilizing HIV type 1 envelope glycoprotein. Proc. Natl. Acad. Sci. USA 1998, 95, 14435-14440

29. Geijtenbeek, T.B.; van Kooyk, Y. Pathogens target DC-SIGN to influence their fate DC-SIGN functions as a pathogen receptor with broad specificity. APMIS 2003, 111, 698-714.

30.Kooyk, Y.V.; Appelmelk, B.; Geijtenbeek, T.B. A fatal attraction:Mycobacterium tuberculosis and HIV-1 target DCSIGN to escape immune surveillance. Trends Mol. Med. 2003, 9, 153-159.

31. Lozach, P.-Y.; Amara, A.; Bartosch, B.; Virelizier, J.-L.; ArenzanaSeisdedos, F.; Cosset, F.-L.; Altmeyer, R. C-type lectins L-SIGN and DC-SIGN capture and transmit infectious hepatitis $\mathrm{C}$ virus pseudotype particles. J. Biol. Chem. 2004, 279, 32035-32045

32. Doores, K.J.; Bonomelli, C.; Harvey, D.J.; Vasiljevic, S.; Dwek, R.A.; Burton, D.R.; Crispin, M.; Scanlan, C.N. Envelope glycans of immunodeficiency virions are almost entirely oligomannose antigens. Proc. Natl. Acad. Sci. USA 2010, 107, 13800-13805.

33. Christopher, G. Selection of a synthetic glycan oligomer from a library of DNA-templated fragments against DC-SIGN and inhibition of HIV gp120 binding to dendritic cells. Chem. Commun. (Camb.) 2011, 47, 9321-9323.

34. Harouse, J.M.; Collman, R.G.; Gonzalez-Scarano, F. Human immunodeficiency virus type 1 infection of SK-N-MC cells: domains of gp120 involved in entry into a CD4-negative, galactosyl ceramide/3'sulfo-galactosyl ceramide-positive cell line. J. Virol. 1995, 69, 7383-7390.

35. Yahi, N.; Baghdiguian, S.; Moreau, H.; Fantini, J. Galactosyl ceramide (or a closely related molecule) is the receptor for human immunodeficiency virus type 1 on human colon epithelial HT29 cells. J. Virol. 1992, 66, 4848-4854.

36. Popik, W.; Alce, T.M.; Au, W.-C. Human immunodeficiency virus type 1 uses lipid raft-colocalized CD4 and chemokine receptors for productive entry into CD4+ T cells. J. Virol. 2002, 76, 4709-4722.

37. Kensinger, R.D.; Catalone, B.J.; Krebs, F.C.; Wigdahl, B.; Schengrund, C.-L. Novel polysulfated galactose-derivatized dendrimers as binding antagonists of human immunodeficiency virus type 1 infection. Antimicrob. Agents Chemother. 2004, 48, 1614-1623.

38. Han, S.; Yoshida, D.; Kanamoto, T.; Nakashima, H.; Uryu, T.; Yoshida, T. Sulfated oligosaccharide cluster with polylysine core scaffold as a new anti-HIV dendrimer. Carbohydr. Polym. 2010, 80, 1111-1115.

39. Han, S.; Kanamoto, T.; Nakashima, H.; Yoshida, T. Synthesis of a new amphiphilic glycodendrimer with antiviral functionality. Carbohydr. Polym. 2012, 90, 1061-1068.

40. Rosa Borges, A.; Wieczorek, L.; Johnson, B.; Benesi, A.J.; Brown B.K.; Kensinger, R.D.; Krebs, F.C.; Wigdahl, B.; Blumenthal, R.; Puri, A. Multivalent dendrimeric compounds containing carbohydrates expressed on immune cells inhibit infection by primary isolates of HIV-1. Virology 2010, 408, 80-88.

41. Dezzutti, C.S.; James, V.N.; Ramos, A.; Sullivan, S.T.; Siddig, A.; Bush, T.J.; Grohskopf, L.A.; Paxton, L.; Subbarao, S.; Hart, C.E. In vitro comparison of topical microbicides for prevention of human immunodeficiency virus type 1 transmission. Antimicrob. Agents Chemother. 2004, 48, 3834-3844.

42. Bhat, S.; Spitalnik, S.L.; Gonzalez-Scarano, F.; Silberberg, D.H Galactosyl ceramide or a derivative is an essential component of the neural receptor for human immunodeficiency virus type 1 envelope glycoprotein gp120. Proc. Natl. Acad. Sci. USA 1991, 88, 7131-7134.

43. Pérez-Anes, A.; Stefaniu, C.; Moog, C.; Majoral, J.-P.; Blanzat, M.; Turrin, C.-O.; Caminade, A.-M.; Rico-Lattes, I. Multivalent catanionic GalCer analogs derived from first generation dendrimeric phosphonic acids. Bioorg. Med. Chem. 2010, 18, 242-248.

44. Kensinger, R.D.; Catalone, B.J.; Krebs, F.C.; Wigdahl, B.; Schengrund, C.-L. Novel polysulfated galactose-derivatized dendrimers as binding antagonists of human immunodeficiency virus type 1 infection. Antimicrob. Agents Chemother. 2004, 48, 1614-1623.

45. Lundquist, J.J. The cluster glycoside effect. Chem Rev. 2002, 102, 555-578.

46. Han, S.; Yoshida, D.; Kanamoto, T.; Nakashima, H.; Uryu, T.; Yoshida, T. Sulfated oligosaccharide cluster with polylysine core scaffold as a new anti-HIV dendrimer. Carbohydr. Polym. 2010, 80, 1111-1115.

47. Katsuraya, K.; Ikushima, N.; Takahashi, N.; Shoji, T.; Nakashima, H.; Yamamoto, N.; Yoshida, T.; Uryu, T. Synthesis of sulfated alkyl malto-and laminara-oligosaccharides with potent inhibitory effects on AIDS virus infection. Carbohydr. Res. 1994, 260, 51-61.

48. Han, S.; Kanamoto, T.; Nakashima, H.; Yoshida, T. Synthesis of a new amphiphilic glycodendrimer with antiviral functionality. Carbohydr. Polym. 2012, 90, 1061-1068.

49. Zhao, H.; Li, J.; Xi, F.; Jiang, L. Polyamidoamine dendrimers inhibit binding of Tat peptide to TAR RNA. FEBS Lett. 2004, $563,241-245$.

50. Wang, W.; Guo, Z.; Chen, Y.; Liu, T.; Jiang, L. Influence of Generation 2-5 of PAMAM Dendrimer on the Inhibition of Tat Peptide/TAR RNA Binding in HIV-1 Transcription. Chem. Biol. Drug. Des. 2006, 68, 314-318.

51. Hamy, F.; Brondani, V.; Flörsheimer, A.; Stark, W.; Blommers, M.J.; Klimkait, T. A new class of HIV-1 Tat antagonist acting through Tat-TAR inhibition. Biochemistry 1998, 37, 50865095. 
52. Zhao, H.; Dai, D.; Li, J.; Chen, Y.; Jiang, L. Quantitative study of HIV-1 Tat peptide and TAR RNA interaction inhibited by poly

53. Asaftei, S.; De Clercq, E. "Viologen" Dendrimers as Antiviral Agents: The effect of charge number and distance. J. Med. Chem. 2010, 53, 3480-3488.

54. Witvrouw, M.; Fikkert, V.; Pluymers, W.; Matthews, B.; Mardel, K.; Schols, D.; Raff, J.; Debyser, Z.; De Clercq, E.; Holan, G. Polyanionic (i.e., polysulfonate) dendrimers can inhibit the replication of human immunodeficiency virus by interfering with both virus adsorption and later steps (reverse transcriptase/integrase) in the virus replicative cycle. Mol. Pharmacol. 2000, 58, 11001108.

55. Vinogradov, S.V.; Poluektova, L.Y.; akarov, E.; Gerson, T.; Senanayake, M.T. Nano-NRTIs: efficient inhibitors of HIV type-1 in macrophages with a reduced mitochondrial toxicity. Antivir. Chem. Chemother. 2010, 21, 1.

56. Buckley, D.L.; Corson, T.W.; Aberle, N.; Crews, C.M. HIV proteasemediated activation of sterically capped proteasome inhibitors and substrates. J. Am. Chem. Soc. 2010, 133, 698-700.

57. Pion, M.; Serramia, M.J.; Diaz, L.; Byszewska, M.; Gallart, T.; García, F.; Gómez, R.; de la Mata, F.J.; Muñoz-Fernandez, M.Á. Phenotype and functional analysis of human monocytes-derived dendritic cells loaded with a carbosilane dendrimer. Biomaterials 2010, 31, 8749-8758.

58. Ionov, M.; Ciepluch, K.; Klajnert, B.; Glińska, S.; Gomez-Ramirez, R.; de la Mata, F.J.; Munoz-Fernandez, M.A.; Bryszewska, M. (allylamine hydrochloride). Biochem. Biophys. Res. Commun. 2003, 312, 351-354.

Complexation of HIV derived peptides with carbosilane dendrimers. Colloids Surf. B Biointerfaces. 2012, 101, 236-242.

59. Rinaldo, C., Dendritic cell-based human immunodeficiency virus vaccine. J. Intern. Med. 2009, 265, 138-158.

60. Rayburn, E.; Wang, W.; Zhang, R.; Wang, H. Antisense approaches in drug discovery and development. In Prog Drug Res.; Springer: Basel, Swizerland, 2005.

61. Chonco, L.; Bermejo-Martín, J.F.; Ortega, P.; Shcharbin, D.; Pedziwiatr, E.; Klajnert, B.; de la Mata, F.J.; Eritja, R.; Gómez, R.; Bryszewska, M. Water-soluble carbosilane dendrimers protect phosphorothioate oligonucleotides from binding to serum proteins. Org. Biomol. Chem. 2007, 5, 1886-1893.

62 Weber, N.; Ortega, P.; Clemente, M.I.; Scharbin, D.; Bryszewska, M.; de la Mata, F.J.; Gómez, R.; Muñoz-Fernández, M. Characterization of carbosilane dendrimers as effective carriers of siRNA to HIV-infected lymphocytes. J. Control Release 2008, 132, 55-64.

63. Zhou, J.; Neff, C.P.; Liu, X.; Zhang, J.; Li H.; Smith, D.D.; Swiderski, P.; Aboellail, T.; Huang, Y.; Du, Q. Systemic administration of combinatorial dsiRNAs via nanoparticles efficiently suppresses HIV-1 infection in humanized mice. Mol. Ther. 2011, 19, 22282238. 